\title{
Experimentally Induced Stress Decreases Ideal Female Reproductive Timing
}

Running title: Experimental Stress Decreases Ideal Female Reproductive Timing \\ ${ }^{1}$ Centre for Comparative and Evolutionary Psychology, Department of Psychology, University of \\ Portsmouth, Portsmouth, UK \\ *Department of Psychology \\ University of Portsmouth \\ Portsmouth \\ Hampshire PO1 2DY \\ United Kingdom \\ Email: abby.chipman@port.ac.uk \\ Telephone: +44 (0)2392846334
}


Previous correlational research shows that childhood adversity is associated with earlier age of reproduction in humans and other species. Such studies, however, cannot show that stressful conditions cause earlier reproduction. Using the cold-pressor task, we built on previous work to test the idea that acute stress influences human reproductive and marital ideals, and that individual stress responses depend on adaptive life history strategies shaped by exposure to adversity during childhood. Acute stress shifted ideal ages of first birth and marriage to earlier ages. We also tested a competing hypothesis, whether stress had a more general impact on time preference, but found no evidence that it did. Furthermore, there was an interaction between childhood adversity and acute stress. Individuals who reported more exposure to childhood adversity responded to acute stress by reporting even earlier reproductive ideals. These findings offer experimental evidence that physiological stress can alter reproductive decision making in humans.

1 Keywords: life history strategy, cold pressor test, acute stress, childhood adversity, female 2 reproductive timing, marital ideals, time preference 


\section{Experimentally induced stress decreases ideal female reproductive timing}

$5 \quad 1.1$ Introduction

6 Like other animals, humans show within-species variation in reproductive behaviour, not least in age

7 of first birth. These adjustments in reproductive timing can be viewed as adaptive behavioural

8 calibrations with the ultimate goal of maximising individuals' reproductive success under particular

9 environmental conditions (Sear et al., 2007). For example, women in high-mortality, resource-poor

10 environments should favour early reproduction in order to bear children before the risk of dying or

11 becoming incapacitated becomes too great. On the other hand, women in low-mortality, resource-rich

12 environments should postpone the onset of reproduction. Through delaying, they give themselves an

13 extended period of somatic investment and resource accumulation that may then be used to produce

14 higher quality offspring (Nettle, 2011). This trade-off in investment that helps an organism to develop

15 its reproductive strategy is know as their life history which falls on a continuum between adopting a

16 fast strategy (earlier age of first birth) or a slow strategy. At its heart these trade-offs are due to

17 allocating limited resources into traits that will maximize fitness. Historically this trade off has been

18 discussed in terms of $r / K$ selection where $r$ selected organisms have more fast life history traits such

19 as short generation times, small body sizes, early maturity and high fecundity. These traits are

20 adaptive in unstable or unpredictable environments with high extrinsic mortality. By contrast, K-

21 selected organisms have long generation times, large body sizes, later maturity and lower fecundity

22 but great offspring survival, traits that are adaptive in stable environments with lower mortality.

23 While selection should favour a point where the costs and benefits of reproductive tradeoffs are

24 optimized, this ultimate approach focuses on the links between particular ecologies and behavioural

25 responses. An equally important approach is the study of the proximate mechanisms that mediate

26 these links. There seem to be various triggers for the onset of early reproduction (Coall and Chisholm,

27 2003; Ellis et al., 2005) in humans. One particular area of interest is the relationship between the

28 onset of reproduction and individual stress responsivity. Stress is a term that has been used loosely in

29 psychology to mean things that are close conceptually but are perhaps not interchangeable such as

30 subjective feelings of being overwhelmed and psychosocial adversity. We focus on the biological

31 usage of stress meaning activation of the hypothalamic-pituitary-adrenal axis, which responds

32 strongly to challenging and uncontrollable threats (Dickerson and Kemeny, 2004). 
33 The stress response system is highly plastic, particularly during early development (Boyce and Ellis,

34 2005; Levine, 2005), and stress is a likely candidate for adjusting individuals' life-history strategies. In

35 non-human species, experimental work has already firmly established the causal link between low

36 early-life investment and altered stress physiology through manipulation of levels of rats maternal

37 grooming and licking of their pups (Bagot et al., 2009). In humans, previous research has firmly

38 established an association between exposure to putative childhood stress and calibrated female life

39 history strategies (Chisholm et al., 2005). This includes stressors related to the family environment

40 during childhood such as father absence (Ellis et al., 2003), levels of parental investment (Belsky et

$41 \mathrm{al}, 1991$ ) and separation of children from their parents (Pesonen et al., 2008). Therefore, in this study

42 we focus specifically on family stress, referring to this as childhood adversity and when using the term

43 stress we mean hypothalamic-pituitary-adrenal axis activation, which has not been established in the

44 case of childhood adversity.

45 The effects of acute stress on life-history strategies in humans are even less well known, although a

46 few experimental studies have begun to examine causal links between physiological stress and

47 reproductive timing. Griskevicius et al. (2011) showed that priming psychological cues of mortality

48 shifts individual fertility intentions. In the case this study, however, the only measures of stress

49 obtained were subjective stress ratings with no evidence of the participants having experienced

50 physiological arousal. As such, the researchers cannot be sure they measured a physiological stress

51 response whether independent or in conjunction with any other psychological or social mechanisms.

52 More explicitly related to physiological stress, exposure to a social stressor demonstrated a

53 relationship between cortisol reactivity and age of first intercourse in females (Brody, 2002). Women

54 with an earlier age of first intercourse (a potential hallmark of a fast life-history strategy) had

55 decreased cortisol levels in response to the stress task compared with women with older ages of first

56 intercourse (possibly on a slower life-history trajectory). Thus it is reasonable to predict that acute

57 stress has the potential to influence an individual's life-history strategy.

58 While we may expect a main effect of stress on reproductive timing, there is also reason to predict an

59 interaction between acute stress and life history trajectory from animal behaviour. Lancaster et al.

60 (2008) looked at reproductive behaviour in female side blotched lizards (Uta stansburiana), which

61 demonstrate both slow and fast life-history strategies. Administering corticosterone (a glucocorticoid 
62 hormone secreted by the adrenal cortex similar to cortisol) to the female lizards delayed reproduction

63 in slow K-strategists, while accelerating reproduction in the fast r-strategists.

64 Previous research suggests that the stress response system is developmentally sensitive and due to

65 changes during maturation will alter resultant behaviour, to match local social and environmental

66 conditions. Del Giudice et al. (2011) proposed the adaptive calibration model, which predicts that

67 responses to stress will depend on the individual's history of adversity. Specifically, those who

68 developed in a dangerous and unpredictable environment should show higher stress responsivity than

69 those who developed in an environment with moderate adversity. We hope to provide more empirical

70 evidence in support of differentiated stress responsivity to acute stressors based on the level of

71 exposure to childhood adversity, which adjust females' life-history trajectories.

72 While previous research has shown that there is an association between childhood adversity and

73 adult behaviour, we hope that by experimentally studying acute stress to add to the understanding of

74 both previous mortality priming studies and previous correlational research. Thus, in this paper, we

75 experimentally tested the effects of acute stress on ideal reproductive timing. We also asked about

76 ideal age of marriage. Although this is not a component of life history theory and specific to humans,

77 variation exists in the timing of marriage (in addition to age of first birth), which typically precedes

78 pregnancy (United Nations Department of Economic and Social Affairs, 2004). As such ideal age of

79 marriage is a potential proxy for entering a life-stage associated with having children. We also

80 investigated the interaction between childhood adversity and acute stress, specifically whether

81 women exposed to different levels of adversity during childhood may react differently to acute stress

82 in terms of adjusting their fertility intentions. If so, this would suggest that exposure to both the

83 repeated stressors of childhood adversity and high stress events could alter women's reproductive

84 timing.

85

$86 \quad 2.1$ Methods and Materials

$87 \quad 2.2$ Participants

88 An opportunity sample of 135 nulliparous and unmarried female undergraduates (mean age 19.84

89 years \pm 3.95 ) was recruited to take part in the study. Participants were required to refrain from 
90 consuming alcohol, caffeine or food for 1 hour before the session so as not to affect cortisol levels.

91 Demographic data for participants can be found in Table 1.

93 2.3. Measures

\subsubsection{Pre-Measures}

Demographic variables were also collected including participants' age, contraceptive use, age of menarche (measured retrospectively) often associated with life history strategy, days since last menstruation, menstrual cycle position and contraceptive use. There were no significant differences between conditions in terms of any demographic variables. Level of exposure to childhood adversity was measured using the 12-item Family Stress Scale (see Appendix A). Answers to each statement were given on a five-point scale where one equalled Strongly Agree and five equalled Strongly Disagree. Higher scores indicate a less stressful family situation (Cronbach's alpha=0.81 and for our sample=0.84) (Mikach and Bailey, 1999). There were no significant differences between conditions for this measure (see Table 2).

105 After the hand immersion participants also completed a free text response to the questions; 'At what age do you want to have your first baby?', 'What would be the ideal number of children you

107 want to have?', and 'At what age do you want to get married?' Participants who did not wish to 108 get married or to have children were instructed to leave the answer blank and were removed 109 from subsequent analysis. Descriptive statistics for these measures are reproduced in Table 2.

111 Individual's stated fertility intentions were used as a proxy measure of actual reproductive 112 scheduling based on Nettle et al.'s (2010) investigation of data from the National Childhood

113 Development Study. This investigation showed that at the onset of adulthood (age 16),

114 individuals have formed conscious intentions regarding their ideal timing of parenthood and 115 women's responses were strong predictors of their actual ages of conception and childbearing 116 even when the event occurred years later. For example, women who wanted to become teenage 
117 mothers were 5.39 times more likely to do so than those who did not. Other longitudinal research

118 has borne out the supposition that fertility intentions serve as consistent predictors of fertility

119 intentions (Schoen et al., 1999). In this study, those who were sure they wanted a child were

120 some 5 to 7 times more likely to have one in the next few years than those who were sure they

121 did not.

\section{$122 \quad$ 2.3.3 Future Discounting}

123 There is also a possibility that any adjustment in fertility intentions may not necessarily specific to

124 reproductive timing, but rather may reflect a more general shift in time preference leading

125 individuals to focus more on present gains and losses, and to discount the future. This

126 alternative hypothesis is supported by experiments which have shown that mortality cues caused

127 future discounting in those who grew up poor (a predictor of fast life history strategies), but had

128 the opposite effect in those who grew up rich (Griskevicius et al., 2011). While childhood

129 socioeconomic status is not the same thing as childhood adversity (socioeconomic status is likely

130 to fall under the adversity umbrella but adversity will also include a wider range of factors such as

131 familial stress) it is still important to determine if any changes to future fertility intentions

132 represent a more general time effect or are sensitive to childhood adversity in the same way that

133 they are to childhood socioeconomic status.

134 In order to test this alternative hypothesis, we measured the discount preference of 63 of the

135 participants (participants were recruited in two blocks, and only the second block were asked

136 these questions). Our measure was based on Chesson et al. (2006) and demonstrates an

137 individual's willingness to forego immediate gratification for future gratification, thus indicating

138 future discounting preference. Discount rates were calculated based on participants' responses

139 to three hypothetical questions about the possible payoff to a prize. In the first question, the

140 participant was asked if they would prefer a prize of $£ 400$ today versus a prize of $£ 1200$ a year

141 from today. In the second and third questions, the future prize was reduced to $£ 800$ and $£ 500$

142 respectively (see Appendix B). From their responses, individuals were categorized from those

143 who strongly favoured delayed gratification (1) to those who strongly favoured immediate

144 gratification (4) (see Table 2 for the descriptive statistics for this measure). There was no

145 significant main effect of condition on discount preference $F(1,60)=1.04, p=.03)$, nor was there 
1492.4 Procedure

150 The socially evaluated cold-pressor or ice-water test is a commonly used and well validated

151 laboratory pain stressor that combines the administration of physiological (cold pressor) and

152 psychological (social-evaluative threat) stressors. This procedure has been shown to activate both the

153 autonomic nervous system and the hypothalamic-pituitary-adrenal axis, with the socially evaluated

154 cold condition has been shown to increase cortisol responses significantly more than the warm

155 condition (Schwabe et al. 2008). This rise in cortisol starts about five minutes after the triggering event

156 with a peak between 10 and 30 minutes.

157 Experimental sessions were run between 14.00 and 17.00 to control for diurnal cycle of cortisol. Each

158 session lasted approximately 30 minutes. When each participant arrived for the experiment, they were

159 seated at a table and chair with their non-dominate hand adjacent to the basin used for the test. After

160 each participant signed the consent form and completed the pre-measure and demographic

161 questions, we attached heart rate monitors and a sphygmomanometer (blood pressure meter) was

162 attached to their dominant arm. Participants' heart rates were recorded for 90 seconds and two

163 baseline blood pressure readings were taken. Next, we randomly assigned participants to the cold

164 water stress condition or a warm water non-stress condition. Due to technical problems during data

165 acquisition, heart rate data for two participants in the ice water condition and five participants in the

166 warm water condition were missing.

$167 \quad 2.4 .1 \quad$ Stress-condition: cold-pressor test

168 The experimenter informed the participants assigned to the stress condition that they would be 169 immersing their hands in ice water for up to three minutes, or for as long as they could tolerate.

170 They were informed that the procedure would potentially be unpleasant and cause discomfort. This 171 information may have added to the psychological stressors experienced by the participants and 172 may account for the pre-measure heart rate being significantly higher in the cold pressor condition $173(t(105)=2.37 p=0.02 r=.23)$. The experimenter asked the participant to insert their non-dominant 
174 hand into the basin of ice water (maintained at $0-4{ }^{\circ} \mathrm{C}$ ) up to their wrist with their palm facing the

175 ceiling. The participants were informed not to make a fist with their hand. Three participants

176 withdrew their hand from the ice water before the three minute limit. The experimenter measured

177 the participants' blood pressure immediately after they immersed their hand in the ice water, and

178 then at one minute intervals until the participant removed their hand from the water. Participants

179 had three blood pressure readings taken at one minute intervals during the ice water hand

180 immersion. There were three participants who removed their hands from the ice water before the

181 three minutes were completed, who had one blood pressure reading taken. Continuous heart rate

182 readings were recorded electronically for each participant at 15 second intervals.

$183 \quad 2.4 .2$ Non-stress condition: warm water test

184 The experimenter informed the participants they would be immersing their hand in water

185 maintained at normal body temperature $\left(35-37^{\circ} \mathrm{C}\right)$ for three minutes. Participants placed their non-

186 dominant hand into the basin of warm water up to their wrist with their palm facing the ceiling. The

187 participants were informed not to make a fist with their hand. Participants kept their hand in the

188 warm water for the full three minutes. Cardiovascular data were recorded as with the cold

189 condition.

190 Immediately after the participants withdrew their hands from the water, the experimenter prompted

191 them to rate separately how stressful, unpleasant and painful the previous hand immersion had been

192 on scales ranging from 0 (not at all) to 100 (very) in 10-point increments. After the subjective ratings

193 were complete, participants' heart rates were measured for 90 seconds and a post-measurement

194 blood pressure reading was taken. Once this was done, the participants completed the fertility

195 intention measures.

1972.5 Statistical Analysis

198 Data were analysed by independent t-tests and univariate general linear model as appropriate with

199 the alpha level set at $p<0.05$. Effect sizes for the significant main and interaction effects are reported

200 as partial $\eta 2$. Following the conventions of Cohen (2013) partial $\eta 2=0.01$ is considered a small effect,

201 partial $\eta 2=0.06$ a medium-sized effect and partial $\eta 2=0.14$ a large effect. 


\section{$203 \quad 3.1$ Results}

204 Physiological and subjective measures indicated that the cold-pressor condition did in fact cause the

205 participants physiological stress in contrast to the control condition. Cardiovascular data showed that 206 participants' systolic $(\mathrm{t}(129)=9.54 p<.001 \mathrm{r}=.64)$ and diastolic $(\mathrm{t}(129)=9.35 p<.001 \mathrm{r}=.64)$ blood

207 pressure readings were significantly elevated in the cold condition only during the hand immersion.

208 Heart rates $(\mathrm{t}(108)=5.05 p<.001 \mathrm{r}=.44)$ were also significantly faster in the cold-pressor condition

209 during the hand immersion and pre hand immersion ( $\mathrm{t}(105)=2.37 p=0.02 \mathrm{r}=.23$ ). Participants in the

210 cold-pressor condition rated the procedure as significantly more stressful $(\mathrm{t}(133)=14.24 p<.001$

$211 \mathrm{r}=.78)$, unpleasant $(\mathrm{t}(133)=28.15 p<.001 \mathrm{r}=.93)$ and painful $(\mathrm{t}(133)=26.92 p<.001 \mathrm{r}=.92)$ than

212 participants in the warm water condition see Table 3.

214 There were significant main effects of acute stress $\left(F(1,120)=15.57, p<.001, \eta^{2}=0.11\right)$ and 215 childhood adversity $\left(F(1,120)=5.85, p=.02, \eta^{2}=0.04\right)$ on participants' reported ideal age of first

216 birth. There were also significant main effects of acute stress $\left(F(1,120)=13.54, p<.001, \eta^{2}=0.09\right)$

217 and childhood adversity $\left(F(1,120)=11.93, p=.001, \eta^{2}=0.08\right)$ on participants' reported ideal age of

218 marriage. For all main effects exposure to acute stress and higher levels of childhood adversity

219 predicted earlier ideal age of marriage for participants. Furthermore, there was a significant interaction

220 between childhood adversity and acute stress on ideal age of first birth $\left(F(1,120)=4.62, p=.03, \eta^{2}=\right.$

$2210.03)$, but no significant interaction for ideal age of marriage $\left(F(1,120)=2.77, p=.10, \eta^{2}=0.02\right)$. This

222 indicates that participants with different levels of exposure to childhood adversity were affected by the

223 cold pressor test differently. Ideal age of first birth was similar in the non-stress condition for

224 participants with more reported childhood adversity $(M=28.69$ years) and less reported childhood

225 adversity ( $M=28.68$ years; participants divided by median split). However in the acute stress condition

226 the ideal age of first birth was lower for participants with more exposure childhood adversity $(M=26.60$

227 years) compared with participants with less exposure $(M=27.77)$. 
229 Results for the main effect of acute stress of ideal age of first birth and marriage are presented in

230 Figure 1 and the interaction effects in Figure 2 and 3 . The cold condition reduced ideal ages of first

231 birth from 28.6 to 27.1 years and ideal age of marriage from 26.8 to 25.4 years. Given that average

232 age was 19.84, the difference equals a $17 \%$ reduction in terms of the time until first birth ([27.1-

$23319.84] /[28.6-19.84]=0.83)$, and the difference in ideal age of marriage a $20 \%$ reduction $([25.4-$

$23419.84] /[26.8-19.84]=0.8)$. These findings suggest that both acute and childhood adversity adjust

235 women's ideal ages of first birth and marriage towards a younger age.

\section{4.1 Discussion}

239 Our findings suggest that both acute stress and childhood adversity (as measured by our response to 240 the family stress scale) play a part in female's reported ideal reproductive timing. We believe this is

241 the first experimental evidence to demonstrate that physiological stress has causal effects on human

242 fertility preferences and provides further support for the impact of childhood adversity on reproductive

243 timing. Our findings suggest that acute stress may be sufficient to shift women's preferences towards

244 early reproduction. Repeated exposures to such stressors during childhood may partly account for the

245 wide variation in age of reproduction within human societies. While the main effect sizes of our main

246 analysis are moderate and the interaction effects are small this is not surprising given that the

247 decision to have children is complex, with many different factors contributing.

248 Using a laboratory stress paradigm to look at the effect of acute stress on ideal reproductive timing we

249 showed a significant effect of stress condition on female's reported age of first birth and marriage to

250 earlier ideals. These findings indicate that the acute stress condition caused women to want to get

251 married and have children sooner compared with participants in the non-stress condition. We also

252 sought to determine whether or not this was a specific effect on reproductive timing or instead a more

253 general effect where female intentions about future reproductive timing are being calibrated through

254 adjusted time preferences for non-fertility decision. Our results do not suggest that acute stress

255 caused a more general effect on female time preference in terms of future discounting. 
256 Finally, we looked at the interaction between acute and childhood adversity and found that women

257 experienced to higher childhood adversity in terms of family stress, responded to acute stress by

258 reporting early ideal age of first birth and marriage compared with those who experience less

259 childhood adversity. These findings are even more striking given that our sample consisted of

260 undergraduate females, who have probably adopted strategies at the slow end of the life-history

261 continuum. That is to say, they have invested in continuing their education as opposed to beginning to

262 reproduce.

263 Results from our studies support the large field of literature suggesting that childhood adversity

264 experienced during development is associated with the direction of an individual's life-history

265 trajectory (Belsky et al., 1991; Bogaert, 2008; Nettle et al., 2011; Pesonen et al., 2008). Furthermore,

266 we corroborate previous work by Griskevicius and colleagues (2011) and provide new experimental

267 evidence that acute stress also has a role to play in regulating female life-history strategies,

268 specifically reproductive timing. While we do not know whether the results of this experiment were due

269 to physiological stress caused hypothalamic-pituitary-adrenal axis activation, it could be that different

270 stressors (psychosocial and physiological) translate into the same physiological currency. It is

271 possible that psychological and physiological stressors work independently, or additively, as

272 suggested by the finding that a psychological threat (social evaluation) increases some effects of the

273 purely physiology cold-pressor test (Schwabe et al., 2008). Future work will be needed to see if

274 physiology alone can account for all effects independent of psychosocial stressors.

275 There may be other ways of interpreting our findings. Wisman and Goldenberg (2005) interpret the

276 effects of mortality salience on fertility preferences in terms of terror management theory, a

277 psychological defence mechanism, rather than life history theory. Theory Management Theory

278 proposes that cultural values (i.e. marriage, childbearing) provide life with meaning and therefore

279 serve to manage the psychological conflict created human's terror of death. Our study may have

280 involved some psychological priming as participants were warned that they might find experiences of

281 the cold pressor condition unpleasant and comfortable. One may also argue that physical pain could

282 still prime mortality implicitly. Ultimately, our data may not distinguish between these two ideas, and

283 they could be considered as proximate and ultimate explanations that are not mutually exclusive. 
284 A question that remains open is how acute stress in the short term might translate to a fast life-history

285 strategy in the long term. We do not suppose that our stress manipulation permanently altered our

286 participants' preferred reproductive timing: most likely any effect soon disappeared and was not

287 permanent. However, frequent and/or severe exposure to stress, perhaps simply the physiological

288 component, may adjust women's psychological preferences. An analogy might be the way a spring is

289 extended by a force. Up until its elastic limit, a spring will return to its original length when the force is

290 removed. But after that point, the spring will undergo plastic deformation, and will remain somewhat

291 extended after the force is removed. In the same way, perhaps women's life-history preferences may

292 be elastic until some limit of stress is reached, at which point they become plastic. Individual

293 differences in where that limit is and how they react after the limit may be analogous to the different

294 elastic limits and plastic deformation curves of different materials.

295 External predictive adaptive response accounts propose that childhood conditions serve as an

296 indicator of the adult environment into which the individual will mature thus making it advantageous for

297 the individual to respond to childhood adversity by developing a reproductive schedule appropriate for

298 that condition. Internal predictive adaptive response account, by contrast, argue that childhood

299 conditions predict the state of an individual's body in adulthood and not the state of their environment

300 (Nettle et al., 2013). Conditions of adversity, therefore, will result in the development of a soma that

301 has a higher risk of mortality at any age and early maturation will increase the individual's chances of

302 achieving some of their reproductive potential. While it is not in the remit of this paper to explore which

303 mechanism most likely results in the earlier maturity and fertility seen in females raised in adverse

304 childhood environments, it is entirely plausible that neither is mutually exclusive that both adaptive

305 responses interact.

306 4.1.2 Conclusion

307 Our findings contribute to the growing field of literature of acute stress and life-history strategies that

308 has not been as extensively tested yet as the relationship of early childhood adversity. However, it is

309 probable that we have mainly tested women who have adopted strategies at the slow end of the life-

310 history continuum as we worked with undergraduates had invested in continued education of bearing

311 children (a typical life-history trade off between future and current reproduction). In order to fully

312 assess how acute stress may potentially adjust reproductive timing ideals and to get a clear picture of 
313 how this would translate to actual fertility behaviour a larger sample with more exposure to different

314 levels of adversity would be useful. It would also be interesting to measure other objective predictors

315 of fast life history strategies such as age of first sexual encounter or number of previous pregnancies

316 or abortions, although we found no baseline differences in age of menarche (another predictor), as

317 expected after random assignment. Furthermore, a more diverse sample would also allow for the

318 study of populations exposed to higher levels of energetic stress, limited resource availability and

319 different socioeconomic status as well as different levels of familial stress who have most likely shifted

320 their strategies towards the fast end of the life history continuum compared with our sample of

321 university undergraduates who are delaying reproduction.

322 Life-history theory predicts that selection should favour a point where the costs and benefits of

323 reproductive trade-offs are optimized. Thus, individuals living in conditions of adversity such as

324 chronic environmental stress who may pay greater costs in delaying childbearing such as the

325 increased risk of death or becoming incapacitated should begin to reproduce earlier. While the links

326 between childhood adversity and reproductive timing have been well studied less is known about how

327 acute stress may also adjust reproductive timing. We show experimentally that, like childhood

328 adversity, acute stress also calibrates women's potential reproductive timing. Furthermore, this effect

329 interacts with childhood adversity in a pattern consistent with our understanding of life-history

330 strategies and the stress response system such that women exposed to more childhood adversity

331 react to acute stress by shifting the ideals to even earlier ages than those not exposed to childhood

332 adversity. We suggest that attention should be paid to acute stressors as well as childhood adversity

333 to increase our understanding of patterns of early reproduction, such as teenage pregnancy. 
335 Bagot, R. C., van Hasselt, F. N., Champagne, D. L., Meaney, M. J., Krugers, H. J., \& Joëls, M. (2009).

336 Maternal care determines rapid effects of stress mediators on synaptic plasticity in adult rat

337 hippocampal dentate gyrus. Neurobiology of learning and memory, 92(3), 292-300.

338 Belsky, J., Steinberg, L., \& Draper, P. (1991). Childhood experience, interpersonal development, and 339 reproductive strategy: An evolutionary theory of socialization. Child development, 62(4), 647-670.

340 (doi:10.2307/1131166)

341 Bogaert, A. F. (2008). Menarche and father absence in a national probability sample. Journal of

342 biosocial science, 40(4), 623. (doi:10.1017/S0021932007002386)

343 Boyce, W. T., \& Ellis, B. J. (2005). Biological sensitivity to context: I. An evolutionary-developmental

344 theory of the origins and functions of stress reactivity. Development and psychopathology, 17(2), 271-

345301.

346 Brody, S. (2002). Age at first intercourse is inversely related to female cortisol stress

347 reactivity. Psychoneuroendocrinology, 27(8), 933-943.

348 Chesson H.W., Leichliter J.S., Zimet G.D., Rosenthal S.L., Bernstein D.I., \& Fife K.H. (2006). Discount

349 rates and risky sexual behaviour among teenagers and young adults. Journal of Risk and Uncertainty

$35032,217-230$.

351 Chisholm, J. S., Quinlivan, J. A., Petersen, R. W., \& Coall, D. A. (2005). Early stress predicts age at

352 menarche and first birth, adult attachment, and expected lifespan. Human Nature, 16(3), 233-265.

353 Coall, D. A., \& Chisholm, J. S. (2003). Evolutionary perspectives on pregnancy: maternal age at 354 menarche and infant birth weight. Social science \& medicine,57(10), 1771-1781.

355 Cohen, J. (2013). Statistical power analysis for the behavioral sciences. Academic press: London

356 Del Giudice, M., Ellis, B. J., \& Shirtcliff, E. A. (2011). The adaptive calibration model of stress

357 responsivity. Neuroscience \& Biobehavioral Reviews, 35(7), 1562-1592.

358 Dickerson, S. S., \& Kemeny, M. E. (2004). Acute stressors and cortisol responses: a theoretical

359 integration and synthesis of laboratory research. Psychological bulletin, 130(3), 355.

360 Ellis, B. J., Bates, J. E., Dodge, K. A., Fergusson, D. M., John Horwood, L., Pettit, G. S., \& Woodward, 361 L. (2003). Does father absence place daughters at special risk for early sexual activity and teenage 362 pregnancy?. Child development, 74(3), 801-821.

363 Ellis, B. J., Essex, M. J., \& Boyce, W. T. (2005). Biological sensitivity to context: II. Empirical 364 explorations of an evolutionary-developmental theory .Development and psychopathology, 17(02), 365 303-328.

366 Griskevicius, V., Delton, A. W., Robertson, T. E., \& Tybur, J. M. (2011). Environmental contingency in 367 life history strategies: the influence of mortality and socioeconomic status on reproductive 368 timing. Journal of personality and social psychology, 100(2), 241. 
369 Lancaster, L. T., Hazard, L. C., Clobert, J., \& Sinervo, B. R. (2008). Corticosterone manipulation

370 reveals differences in hierarchical organization of multidimensional reproductive trade-offs in

371 r-strategist and K-strategist females. Journal of evolutionary biology, 21(2), 556-565.

372 Levine, S. (2005). Developmental determinants of sensitivity and resistance to

373 stress. Psychoneuroendocrinology, 30(10), 939-946.

374 Mikach, S. M., \& Bailey, J. M. (1999). What distinguishes women with unusually high numbers of sex 375 partners? Evolution and Human Behavior, 20(3), 141-150.

376 Nettle, D. (2011). Flexibility in reproductive timing in humans: Integrating ultimate and proximate 377 explanations. Philosophical Transactions of the Royal Society, B., 366, 357-65.

378 Nettle, D., Coall, D. A., \& Dickins, T. E. (2010). Early-life conditions and age at first pregnancy in 379 British women. Proceedings of the Royal Society B: Biological Sciences, 278(1712), 1721-1727.

380 Nettle, D., Frankenhuis, W. E., \& Rickard, I. J. (2013). The evolution of predictive adaptive responses 381 in human life-history. Proceedings of the Royal Society B: Biological Sciences, 280(1766), 20131343.

382 Pesonen, A. K., Räikkönen, K., Heinonen, K., Kajantie, E., Forsén, T., \& Eriksson, J. G. (2008).

383 Reproductive traits following a parent-child separation trauma during childhood: a natural experiment

384 during World War II. American Journal of Human Biology, 20(3), 345-351. (doi:10.1002/ajhb.20735)

385 Schoen, R., Astone, N. M., Kim, Y. J., Nathanson, C. A., \& Fields, J. M. (1999). Do fertility intentions 386 affect fertility behavior?. Journal of Marriage and the Family, 790-799.

387 Schwabe, L., Haddad, L., \& Schachinger, H. (2008). HPA axis activation by a socially evaluated cold388 pressor test. Psychoneuroendocrinology, 33(6), 890-895.

389 Sear, R., Lawson, D. W., \& Dickins, T. E. (2007). Synthesis in the human evolutionary behavioural 390 sciences. Journal of evolutionary psychology, 5(1), 3-28. (doi:10.1556/JEP.2007.1019)

391 United Nations Department of Economic and Social Affairs. (2004). World population monitoring 2002: 392 Reproductive rights and reproductive health. New York: Department for Economic and Social Affairs.

393 Wisman, A., \& Goldenberg, J. L. (2005). From the grave to the cradle: Evidence that mortality salience 394 engenders a desire for offspring. Journal of Personality and Social Psychology, 89(1), 46. 


\section{Appendix A: Family Stress Scale}

397 1. I have happy memories of my childhood

398 2. My father was always there when I needed him

399 3. I often heard my parents fighting*

400 4. I would like my future relationship with my mate to be like my parents

4015 . I remember doing activities with my family

402 6. I want to raise my children in the same manner my parents raised me

403 7. My parents openly displayed their affection for each other

404 8. I never saw my father hit my mother

405 9. My mother was always there when I needed her

406 10. I wanted to, or did, run away from home*

407 11. When I needed help, my parents were there for me

408 12. My father always seemed to care about what I did when I was growing up

$409{ }^{*}$ Reverse Scored

410

\section{Appendix B: Future Discount Measure}

412 1. I would rather get $£ 400$ today than $£ 1200$ one year from today

413 2. I would rather get $£ 400$ today than $£ 800$ one year from today

4143 . I would rather get $£ 400$ today than $£ 500$ one year from today 
415 Table 1 Descriptive statistics for demographic variables for participants in the warm water and cold416 pressor conditions. Data are means (SE) unless otherwise stated.

\begin{tabular}{lcc}
\hline & $\begin{array}{c}\text { Warm water condition } \\
(\mathbf{n}=\mathbf{6 8})\end{array}$ & $\begin{array}{c}\text { Cold-pressor condition } \\
(\mathbf{n}=\mathbf{6 7})\end{array}$ \\
\hline Age (range 18 to 31) & $19.86(0.44)$ & $19.88(0.55)$ \\
$\begin{array}{l}\text { Contraceptive use } \\
\text { (Count of women using contraception) }\end{array}$ & 36 & 42 \\
$\begin{array}{l}\text { Menstrual cycle stage } \\
\text { (Count of women on luteal stage) }\end{array}$ & 34 & 33 \\
Age of Menarche (range 10 to 16) & $12.94(0.15)$ & $12.88(0.17)$ \\
\hline
\end{tabular}

417

418 Table 2 Descriptive statistics for the Family Stress Scale and the main dependent variables

\begin{tabular}{lcc}
\hline & Range & Mean (SE) \\
\hline Family Stress Scale Scores & -32 to 46 & $18.42(1.44)$ \\
Discount Preference & 1 to 7 & $3.77(0.23)$ \\
Ideal Age of First Birth & 19 to 35 & $27.96(0.23)$ \\
Ideal Age of Marriage & 20 to 32 & $26.21(0.19)$ \\
Desired Number of Children & 0 to 4 & $2.38(0.08)$ \\
\hline
\end{tabular}

419 
420 Table 3 Mean (SE) subjective stress ratings and cardiovascular measures before (pre), during and 421 after (post) hand immersion in the warm water or cold water conditions

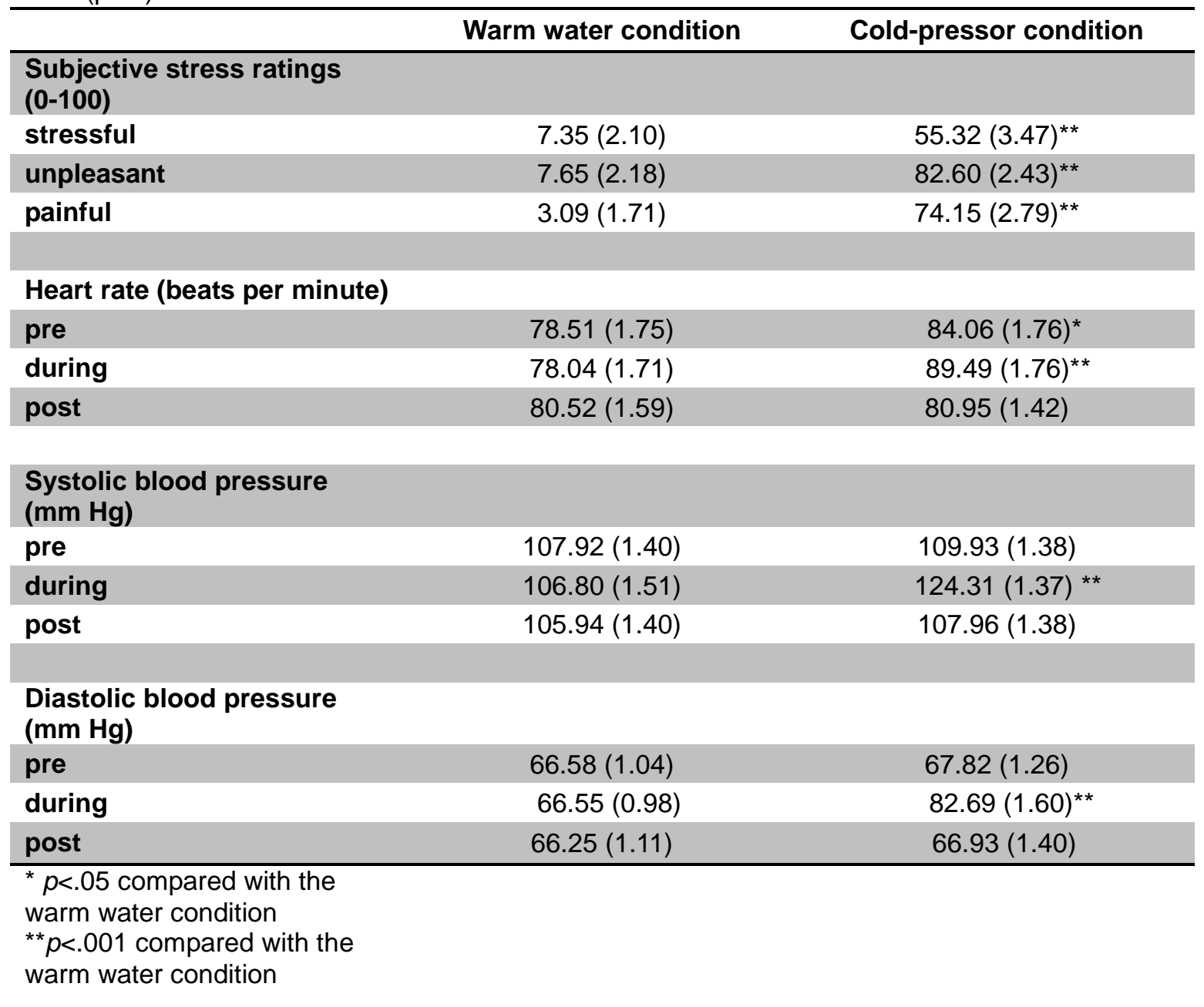




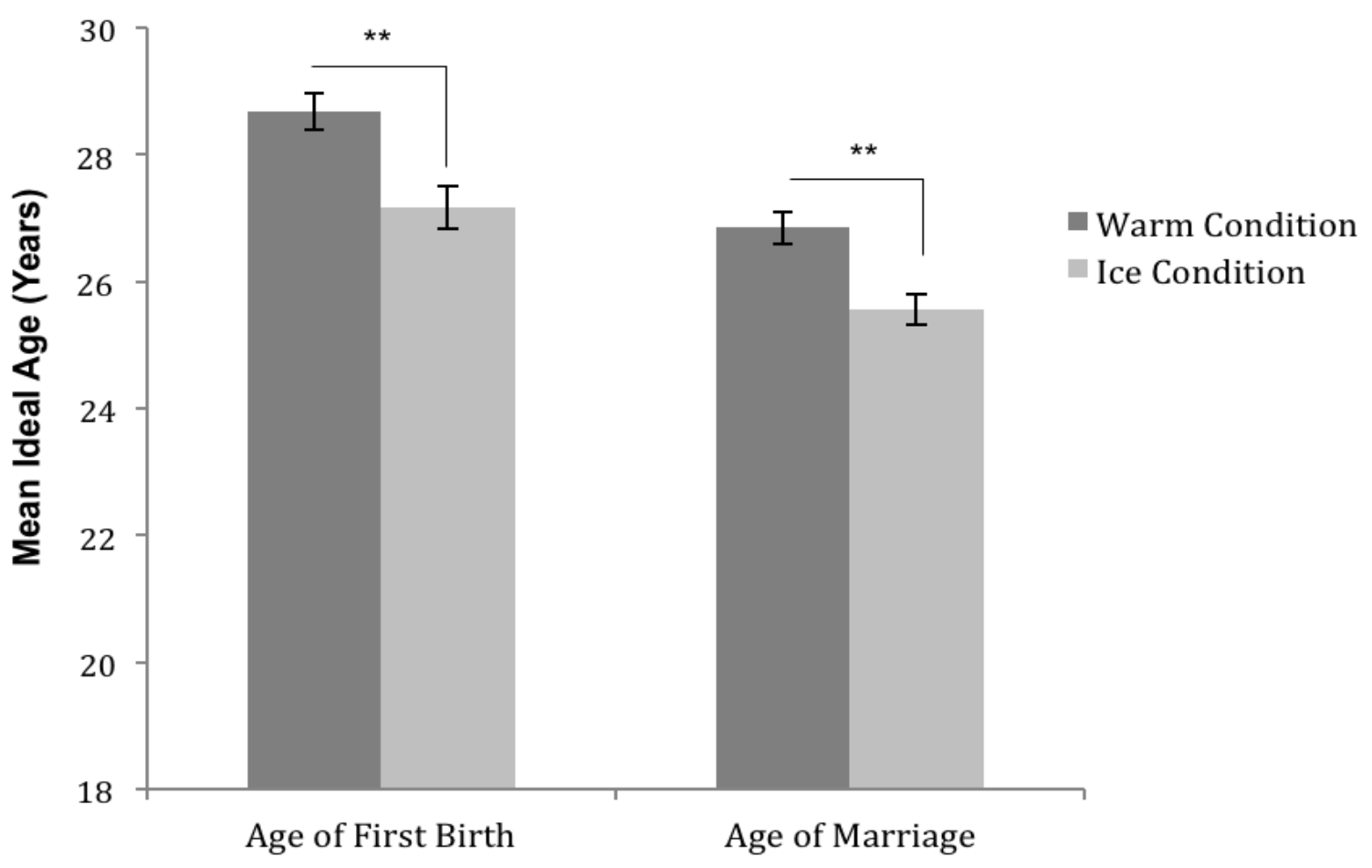

423 Figure 1 Mean (SE) ideal age of first birth and ideal age of marriage. Dark bars designate warm water 425 condition; light bars designate cold-pressor condition.

$426^{* *} p<0.001$

427 


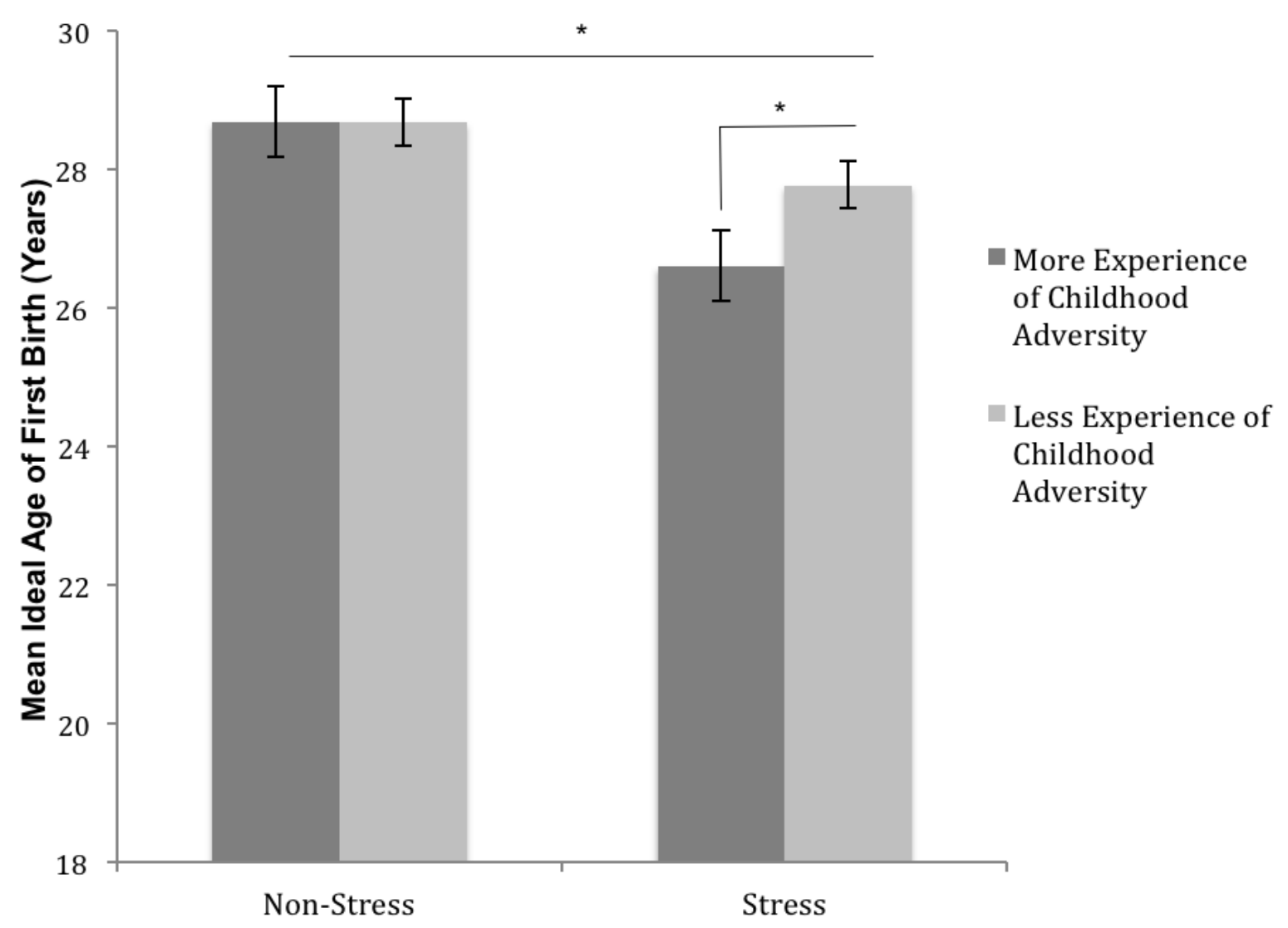

428 Figure 2 Mean (SE) ideal age of first birth by acute stress condition and experience of childhood

430 adversity (CA). CA has been divided by median split. Dark bars designate more experience of CA;

431 light bars designate less experience of CA.

$432 * p<0.05$

433 


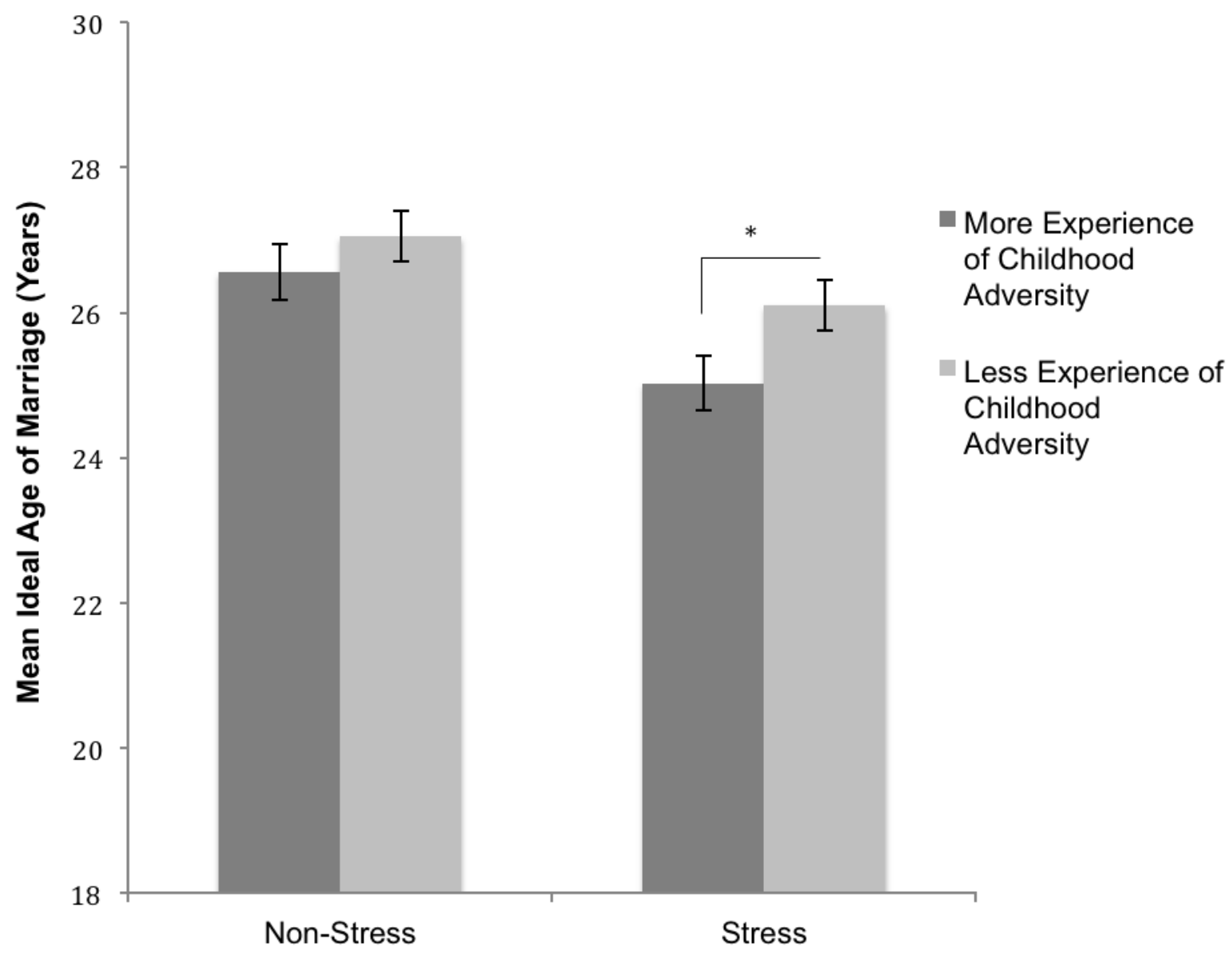

434

435 Figure 3 Mean (SE) ideal age of marriage by acute stress condition and experience of childhood

436 adversity (CA). CA has been divided by median split. Dark bars designate more experience of CA;

437 light bars designate less experience of CA.

$438{ }^{*} p<0.05$ 